\title{
Recent progress in first-principles studies of magnetoelectric multiferroics
}

\author{
Claude Ederer, Nicola A. Spaldin \\ Materials Department, University of California, Santa Barbara, CA 93106
}

\begin{abstract}
Materials that combine magnetic and ferroelectric properties have generated increasing interest over the last few years, due to both their diverse properties and their potential utility in new types of magnetoelectric device applications. In this review we discuss recent progress in the study of such magnetoelectric multiferroics which has been achieved using computational first-principles methods based on density functional theory. In particular, we show how first-principles methods have been successfully used to explain various properties of multiferroic materials and to predict novel effects and new systems that exhibit multiferroic properties.
\end{abstract}

Key words: multiferroics, first-principles calculations, magnetoelectric PACS: 71.15.Mb, 75.30.-m, 77.80.-e

\section{Introduction}

Magnetoelectric multiferroics are materials which exhibit both magnetic order and ferroelectricity in the same phase. Such materials, although rare, have been known since the 1960s and various early review articles systematically classified their properties and behavior $[1,2,3,4]$. In the last few years the field of multiferroic materials has seen a tremendous boom, initiated in part by computational first-principles studies explaining the basic physics underlying their scarcity [5]. The subsequent flurry of research activity led to the discovery of several new multiferroic materials, as well as the identification of various strong coupling effects between their magnetic and ferroelectric degrees of freedom $[6,7,8,9,10,11]$. The prospect of using these coupling effects for new

Email addresses: ederer@mrl.ucsb.edu (Claude Ederer), nicola@mrl.ucsb.edu (Nicola A. Spaldin). 
types of devices in microelectronics and digital memory applications has in turn spawned further interest.

This newer work on magnetoelectric multiferroics was recently reviewed in Ref. [12], with an emphasis on experimental and technological studies. The purpose of the present article is to provide an update on the recent activities in first-principles studies of magnetoelectric multiferroics, with the goal of illustrating the utility of these methods both in explaining unusual behavior in existing multiferroics, and in predicting new materials and novel effects. An extensive review of density functional theory-based studies of multiferroic materials before 2002 was given in Ref. [13]. Since then, first-principles studies of multiferroic materials have made considerable progress; here we summarize these recent developments and point out possible directions for future work.

Ref. [13] reviewed two major questions which were successfully answered by first-principles electronic structure calculations. The first question, "Why are there so few magnetoelectric multiferroics?" [5] is now understood to result from a chemical incompatibility between magnetism and conventional ferroelectricity. This incompatibility is related to the fact that the most common mechanism for ferroelectricity in perovskite-structure oxides involves the presence of a transition metal cation on the perovskite $B$ site with a formal $d^{0}$ electron configuration. In fact, first-principles electronic structure calculations have played a major role in elucidating the role of the $d^{0}$ electron configuration in the ferroelectric instability in perovskite oxides $[14,15,16]$. On the other hand, for magnetism to occur in such transition metal oxides a partially filled $d$ shell is indispensable. The existence of at least some known materials where magnetism and ferroelectricity coexist then poses the second question addressed in Ref. [13]: "Why are there any magnetoelectric multiferroics?" [17]. Clearly, if the ferroelectricity in a multiferroic is caused by a $d^{0}$ cation, a different cation is needed to introduce the magnetism; alternatively, if only magnetic cations with partially filled $d$ shells are present, a new mechanism for ferroelectricity is required. To our knowledge, all known multiferroics adopt the latter scenario. An alternative mechanism for ferroelectric "off-centering", which was discussed in Ref. [13], is the stereochemically active lone pair in cations such as $\mathrm{Pb}^{2+}$ or $\mathrm{Bi}^{3+}$. Again, first-principles calculations were invaluable in showing that this mechanism is responsible for the ferroelectricity in multiferroics such as $\mathrm{BiMnO}_{3}$ or $\mathrm{BiFeO}_{3}[18,19]$. In these systems, the $\mathrm{Bi}^{3+}$ cation has a formal valence electron configuration of $6 s^{2} 6 p^{0}$ and the energy of the system can be lowered by off-centering of the $\mathrm{Bi}$ ion with respect to its oxygen surrounding, which leads to a hybridization of both $\mathrm{Bi} 6 s$ and $6 p$ states with $\mathrm{O} 2 p$ orbitals and a localization of the lone pair on one side of the Bi ion [18].

The organization of this article is as follows. In the following section we give a brief overview of some methodological aspects of first-principles electronic 
structure calculations that are especially relevant to the study of magnetoelectric multiferroics. Some recent methodological developments have contributed to the current boom in magnetoelectric multiferroics, since they facilitate a realistic description of the electronic structure of multiferroic materials and allow the calculation of quantities that are difficult to access experimentally.

Following this brief methodological part, we discuss how first-principles calculation have led to substantial progress in the understanding of multiferroic materials during the last few years. We begin with cases in which first-principles calculations have explained properties of existing multiferroics. As examples we discuss the identification of the mechanism responsible for ferroelectricity in the hexagonal manganite $\mathrm{YMnO}_{3}$, a material which is representative for a whole class of multiferroics, as well as the resolution of controversy surrounding confusing and contradictory experimental data in multiferroic $\mathrm{BiFeO}_{3}$. Following that, we show how first-principles calculations have made important predictions in advance of their experimental observation. Here, we also discuss two examples: The identification of a mechanism for coupling between magnetic and ferroelectric order parameters that could facilitate the intriguing effect of electric-field induced magnetization switching, and the ab initio design of new multiferroic materials with superior materials properties. Finally, we give an outlook on future research in the field, and point out where first-principles methods can further contribute to the study of magnetoelectric multiferroics.

\section{Computational tools}

In this section we briefly summarize recent developments in electronic structure methods which are particularly important for, and in one case were motivated by, the study of multiferroic systems. For a complete description of density-functional-theory-based first-principles methods we direct the reader to one of the excellent texts which are available, e.g. Refs. [20,21].

The first electronic structure calculations of multiferroic systems were performed using the local spin density approximation (LSDA) to density functional theory [20]. Although the LSDA is a well-established technique that continues to make invaluable contributions to our understanding of ferroelectrics (as well as many other materials), it has a number of limitations, among them its well-known underestimation of the size of the band gap for most insulating materials. For systems containing localized electrons with strong Coulomb correlations such as transition metal oxides the use of the LSDA can be totally inadequate, since it can lead to metallic solutions for systems that are known experimentally to be insulators [22]. In the case of magnetic ferroelectrics this is particularly problematic, since it prohibits the calculation of 
the spontaneous ferroelectric polarization and other indicators for structural instabilities, such as Born effective charges [23]. Therefore, the most important recent progress in first-principles methodology, with respect to the study of multiferroic materials, has been the development of methods that facilitate a realistic description of the electronic structure of magnetic systems containing strongly localized $d$ or $f$ electrons.

Two methods that attempt to cure some of the deficiencies of the LSDA in the treatment of localized electrons were developed recently and have been applied to study multiferroic materials: the so-called "LSDA plus Hubbard $U$ " method $(\mathrm{LSDA}+U)[24]$ and a self-interaction corrected pseudopotential method ("pseudo-SIC") [25]. Although the physical motivation behind these two methods is different, they lead to very similar results in practice.

The development of the pseudo-SIC method was actually motivated by the inability of the LSDA to produce a band gap for multiferroic $\mathrm{YMnO}_{3}$ (see section 3.1 below). In general, self-interaction corrected (SIC) methods are based on the well-known fact that the failures of the LSDA can be, at least in part, attributed to the presence of the self-interaction (SI) in the LSDA energy functional, that is the interaction of an electron charge with the Coulomb and exchange-correlation potential generated by the same electron [20]. The SI is strong for spatially localized electrons such as $3 d$ and $4 f$ electrons, and leads to an underestimation of the binding energies, on-site Coulomb energies and exchange splittings of the corresponding states, while the hybridizations of cation $d$ and anion $p$ states and the corresponding band widths are usually overestimated. Possible strategies for eliminating the SI in density functional theory are long-standing issues, but often require a large computing effort even for materials with small unit cells. In the pseudo-SIC method [25], the SI correction is calculated for the corresponding atoms (as in Ref. [26]), and is then scaled by the electron occupation numbers of specific atomic-like orbitals calculated self-consistently within the crystal environment. This approach can be efficiently implemented within a standard pseudopotential method and allows the SI originating from localized, hybridized, or completely itinerant electrons to be discriminated, which permits the treatment of insulating as well as metallic compounds, with minimal computational overhead beyond the LSDA [25].

The motivation behind the development of the LSDA $+U$ method is somewhat different, although in practice this method leads to very similar results as the pseudo-SIC method. In the LSDA $+U$ method the Coulomb interaction between specific localized orbitals is explicitly taken into account by adding a term to the total energy that resembles the well-known "Hubbard Hamiltonian" used to describe Mott-Hubbard metal-insulator transitions [27]. The resulting potential leads to an energy splitting between occupied and unoccupied states. The Hubbard parameter, $U$, which describes the strength of the 
screened electron-electron interaction, can in principle be calculated ab initio but in practice it is often treated as an empirical parameter. Simultaneously, a "double-counting correction" is subtracted from the total energy in order to account for those interaction effects between the localized electrons that are already included in the conventional LSDA. Different forms for this correction term are in use, some of which are in part also designed to make the resulting energy functional exhibit certain desirable properties [28]. These ambiguities and the partly empirical construction of the Hubbard correction is somewhat unsatisfying and the resulting method is strictly speaking not purely first principles. Nevertheless, the LSDA $+U$ method is nowadays used more and more routinely in electronic structure calculations for $d$ and $f$ electron systems and has undoubtedly led to considerable progress in the field.

Simultaneously with the tremendous improvements in the first-principles description of magnetic insulators provided by the LSDA+U and pseudo-SIC methods, the so-called "modern theory of polarization" has revolutionized the first-principles study of ferroelectric materials [29,30,31]. This theory provides both conceptual understanding of the spontaneous polarization in bulk crystals, and computational tools to calculate it from the Kohn-Sham wavefunctions. Complications in calculating the electric polarization in bulk crystals arise from the periodic boundary conditions employed in most solid state calculations. This periodicity leads to an ambiguity in the value of the polarization modulo an integer multiple of the so called "polarization quantum", $\frac{e R}{V}$, such that

$$
P=P^{\prime}+n \frac{e R}{V},
$$

where $P$ and $P^{\prime}$ are two allowed values of the polarization, $n$ is an integer number, $e$ is the electronic charge, $V$ is the unit cell volume, and $R$ is a primitive lattice vector of the underlying Bravais lattice. The polarization of an infinite periodic crystal is therefore represented by a lattice of values, the socalled "polarization lattice". In an experiment only polarization changes can be measured and the corresponding value has to be calculated as a difference between two points of the polarization lattices corresponding to the initial and final states of the system. The corresponding change in polarization is uniquely defined as long as the system stays insulating for all intermediate states transforming the initial to the final state.

Figure 1 shows the evolution of the polarization lattice in multiferroic $\mathrm{BiFeO}_{3}$ calculated for different points along a ferroelectric switching path leading from the negatively polarized ferroelectric state $(-100 \%$ distortion), through the corresponding centrosymmetric structure ( $0 \%$ distortion), to the positively polarized state $(+100 \%$ distortion) [19]. The vertical columns of black circles represent various points of the polarization lattice for a fixed amount of distortion, separated by the polarization quantum of $185.6 \mu \mathrm{C} / \mathrm{cm}^{2}$. The dashed lines show how the polarization lattice changes along the path. 


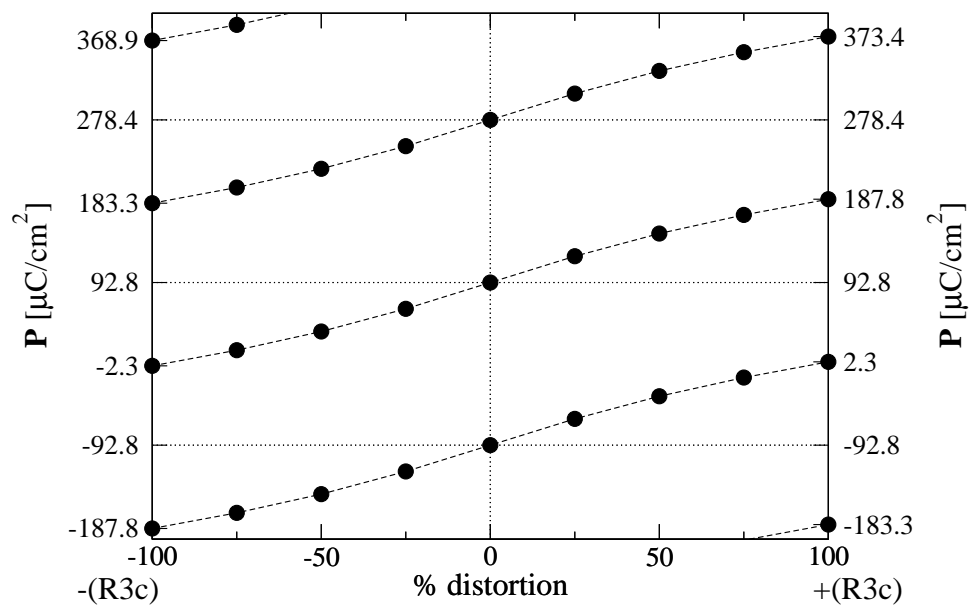

Fig. 1. Change in polarization $P$ along a ferroelectric switching path for multiferroic $\mathrm{BiFeO}_{3}$. The possible values of $P$ for fixed distortion differ by multiples of the polarization quantum, here $185.6 \mu \mathrm{C} / \mathrm{cm}^{2}$. From Ref. [19]. Copyright (2005) by the American Physical Society.

The polarization lattice can be calculated as a gauge invariant geometric phase of the wave-functions, the so-called "Berry-phase" [29,30,31]. This expression solves the problem arising from the unboundedness of the quantum mechanical position operator with the infinitely extended periodic wave-functions in the solid and is compatible with the "multivaluedness" of the polarization; the ambiguity of the polarization modulo a quantum translates into an ambiguity of the Berry-phase modulo integer multiples of $2 \pi$.

In addition to "beyond LSDA" methods like pseudo-SIC and LSDA $+U$, which result in stable insulating solutions for most magnetic oxides, and the modern theory of polarization, which allows an accurate calculation of experimentally measurable polarization differences, there are many other developments in first-principles-based methods that are relevant to the study of multiferroic materials, which we mention briefly. For example, many multiferroic systems have complex noncollinear spin structures, and coupling effects between the polar and magnetic degrees of freedom can be due to spin-orbit coupling. Therefore, a fully noncollinear treatment of the spinor wave-functions and the inclusion of spin-orbit coupling in the calculations is essential to describe many of the interesting properties of multiferroic materials. Also, the analysis of ferroelectric instabilities using calculated phonon spectra is a very powerful tool to explain the appearance of multiferroic properties and predict the existence (or absence) of such instabilities in designing new magnetoelectric multiferroic materials. 


\section{First-principles explanation of experimental observations}

We now outline recent first-principles work that has led to substantial progress in the understanding of known multiferroic materials. First we describe studies aimed at understanding the unusual mechanism for ferroelectricity in the hexagonal manganite materials. Then we discuss a range of seemingly contradictory experimental reports of both the magnetic and ferroelectric data in multiferroic $\mathrm{BiFeO}_{3}$, and show how first-principles calculations were able to resolve some of the controversy.

\subsection{Elucidating the origin of ferroelectricity in hexagonal manganites}

Hexagonal $\mathrm{YMnO}_{3}$ is the prototype of the class of isostructural multiferroic materials with the chemical formula $R \mathrm{MnO}_{3}(R=\mathrm{Ho}-\mathrm{Lu}, \mathrm{Y})$ and room temperature space group $P 6_{3} \mathrm{~cm}$. These materials are ferroelectric with high ferroelectric Curie temperatures of $\sim 1000 \mathrm{~K}$, and exhibit noncollinear antiferromagnetic order below $T_{N} \sim 100 \mathrm{~K}$. Although there have been many recent experimental studies of the corresponding rare-earth systems, primarily $\mathrm{HoMnO}_{3}$, which show a complicated phase diagram at low temperatures, the theoretical first-principles work has focused on $\mathrm{YMnO}_{3}$, partly because of the absence of $4 f$ electrons in this system, which are difficult to treat using pseudopotential-based methods. The main effort has been directed to understanding the mechanism for the occurrence of ferroelectricity, which is clearly of an unconventional origin, since the $\mathrm{Mn}^{3+}$ cation does not have a $d^{0}$ electron configuration and $\mathrm{Y}^{3+}$ does not have a lone pair, so the system contains no "ferroelectrically active" ions. Initial progress in understanding the ferroelectric behavior was hampered by the severe underestimation of the gap within LSDA [16], and by the fact that early experiments incorrectly reported an offcentering of the Mn ions from the centers of their polyhedra in the ferroelectric phase [32].

The first important contribution from first-principles calculations was the analysis of the electronic structure of hexagonal $\mathrm{YMnO}_{3}$ using the LMTOASA method [33]. These calculations, using the early experimentally reported paraelectric and ferroelectric structures, showed that the $\mathrm{Mn}^{4+}$ ion in hexagonal $\mathrm{YMnO}_{3}$ is not a Jahn-Teller ion despite of its $d^{4}$ electronic configuration, because the trigonal crystal field splitting in this material does not lead to an orbital degeneracy [33]. The width of the band gap was shown to be very sensitive to the magnetic ordering, increasing (within the LSDA) from about $0 \mathrm{eV}$ for ferromagnetic [33] and A-type antiferromagnetic [34] order to $0.47 \mathrm{eV}$ for a more complicated but still collinear antiferromagnetic spin arrangement [33]. The width of the gap was also shown to strongly increase if correlations 
were incorporated in the calculations through an LSDA $+U$ treatment $[34,33]$. This led to the conclusion that both magnetic ordering and correlation effects have a strong influence on the electronic structure, and play an important role for the gap formation in hexagonal $\mathrm{YMnO}_{3}$. These calculations also showed, however, that the overall electronic structure is not strongly affected by the experimentally reported structural distortions leading from the high temperature paraelectric phase to the low-temperature ferroelectric phase. The only significant change observed in the calculations was a slightly stronger $p$ - $d$ hybridization due to the off-center displacement of the Mn ion within its surrounding oxygen cage that was reported in the early experiments [32].

Subsequent plane-wave pseudopotential calculations confirmed that such displacements of the Mn cation along the hexagonal axis indeed lead to a certain degree of rehybridization between $\mathrm{O} 2 p$ and the Mn $3 d_{z^{2}}$ orbitals which are oriented along the polar $c$ direction, whereas displacements within the $c$ plane are not accompanied by large changes in chemical bonding $[35,16]$. These observations, together with the experimental reports of $\mathrm{Mn} c$-axis displacements, led to the suggestion that a "one-dimensional $d^{0}$-ness", i.e. the unoccupied nondegenerate $d_{z^{2}}$ orbital oriented along the displacement direction, could be responsible for the stabilization of the ferroelectric state [16]. Nevertheless, an energy-lowering due to the displacement of the Mn ions was not found in the first-principles calculations. It was unclear at this stage whether the absence of energy lowering was an artifact of the use of the LSDA, which does not lead to an appropriate description of the electronic structure of $\mathrm{YMnO}_{3}$ (in fact, in the plane-wave pseudopotential calculations the system remained metallic even in the case of A-type antiferromagnetic ordering), or an indicator that the early experiments reporting a Mn $c$-axis displacement were incorrect. Therefore, a conclusive picture of the ferroelectricity could not be achieved.

The first breakthrough came through a collaboration that combined progress on both the experimental and the theoretical side. First, new single crystal x-ray diffraction experiments, using reflections of the entire Ewald sphere, did not find an off-centering of the Mn ions within their oxygen cages [36]. Instead, the experimental data indicated that the Mn ions remain in the center of their oxygen polyhedra which instead undergo a collective rotation that leads to unit cell tripling. In addition, the $\mathrm{Y}$ ions move along the hexagonal $c$ axis, and fill the space that is freed due to the rotation of the oxygen bipyramids (see Figure 2). Second, on the theoretical side the development of the self-interaction corrected pseudopotential (pseudo-SIC) method [25], motivated by the inadequacies of the LSDA, enabled a full structural optimization of $\mathrm{YMnO}_{3}$ while giving a realistic description of the underlying electronic structure. These calculations confirmed the new experimental data, giving structural parameters very close to those obtained experimentally. The first-principles calculations also showed that the structural distortions in the low temperature phase do not lead to any significant charge redistribution. This is reflected in the values 

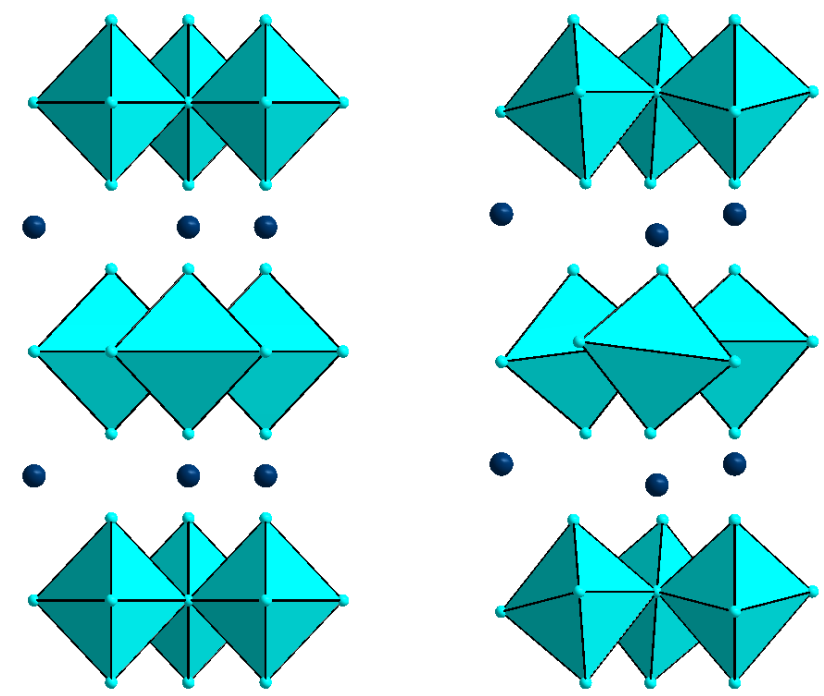

Fig. 2. High temperature centrosymmetric (left) and low-temperature ferroelectric structure (right) of $\mathrm{YMnO}_{3}$. In the low-temperature structure the oxygen polyhedra (turquoise) undergo a collective rotation that leads to a unit cell tripling. This unit cell tripling is accompanied by displacements of the $\mathrm{Y}$ cations (blue) along the (0001) directions which lead to an electric dipole moment.

of the Born effective charges, which were found to be very close to the formal charges. It became clear that rehybridization is not responsible for stabilizing the ferroelectric state in $\mathrm{YMnO}_{3}$ and that in fact the interplay between polar and nonpolar structural modes is essential to the ferroelectricity in this system [37].

A concise picture of this interplay between polar and nonpolar structural modes was obtained very recently by calculating and analyzing the phonon instabilities in the high temperature centrosymmetric structure [38]. It was shown that the only unstable phonon mode is a unit-cell tripling mode, which results in the experimentally observed collective tilting of the oxygen polyhedra and a buckling of the Y (0001) planes ( $K_{3}$ mode). Although this mode on its own does not lead to an electric dipole moment, it reduces the symmetry of the system to the polar space group $P 6_{3} \mathrm{~cm}$, which corresponds to the experimentally observed low-temperature structure. If the distortions that lead from the high-temperature centrosymmetric to the low-temperature ferroelectric phase are decomposed into contributions from different phonon modes, the contribution of this $K_{3}$ mode makes up for more than $80 \%$ of the final distortion. The second largest contribution (about $15 \%$ ) is due to a zone-center polar mode $\left(\Gamma_{2}^{-}\right.$mode) which is compatible with the symmetry reduction due to the unstable $K_{3}$ mode but is stable in the centrosymmetric high-temperature structure. By mapping out the energy surface around the prototype phase as a function of the $K_{3}$ and the polar $\Gamma_{2}^{-}$mode it was shown that the polar mode remains stable for all values of $K_{3}$ but that due to a symmetry allowed coupling term between the $\Gamma_{2}^{-}$and $K_{3}$ modes the equilibrium value of the $\Gamma_{2}^{-}$ 
distortion amplitude is shifted to a nonzero value. The $K_{3}$ mode therefore acts as a "geometric field" on the $\Gamma_{2}^{-}$mode. This behavior is indicative of an $\mathrm{im}$ proper ferroelectric where the polar mode is not the primary order parameter and this mechanism has been suggested to be the driving force behind the ferroelectricity in $\mathrm{YMnO}_{3}[38]$.

The example of $\mathrm{YMnO}_{3}$ shows how first-principles calculations of phonon spectra and analysis of the calculated electronic structure can give a good explanation of the mechanism driving the ferroelectric phase transition in multiferroic materials. It is also apparent that the use of the $\mathrm{LSDA}+U$ and pseudo-SIC "beyond LDA" methods, and the resulting realistic description of the electronic structure, has been essential for such progress to be achieved.

\subsection{Resolving the true magnetization and polarization in $\mathrm{BiFeO}_{3}$}

The determination of the spontaneous polarization and magnetization in $\mathrm{BiFeO}_{3}$ is an example of how valuable first-principles calculations are for determining intrinsic material properties and explaining contradictory experimental data. In a real sample, effects such as epitaxial strain, defects, microstructure, or interface properties can alter the physical properties of a material. Therefore it is sometimes extremely difficult to determine the intrinsic material properties unambiguously using only experimental data. First-principles calculations on the other hand can deliver reference data corresponding to a perfect sample containing absolutely no defects. After establishing the intrinsic value of a certain quantity, these calculations can also be used to determine how effects such as epitaxial strain, various kinds of defects, or the presence of interfaces and surfaces change this intrinsic value.

Perovskite-structure $\mathrm{BiFeO}_{3}$ is one rare example of a magnetoelectric multiferroic that exhibits both magnetic and ferroelectric ordering above room temperature. The bulk material becomes ferroelectric below $T_{C} \approx 1103 \mathrm{~K}$ [39], adopting the $R 3 c$ structure shown in Fig. 3. The magnetic moments of the Fe cations order antiferromagnetically (G-type) below $T_{N} \approx 643 \mathrm{~K}[40,41]$, and in addition it exhibits a long-wavelength spiral-spin structure with a wavelength of about $620 \AA$ [42]. Until recently, the value of the polarization in $\mathrm{BiFeO}_{3}$ was believed to be rather small. The only current-voltage measurements performed on $\mathrm{BiFeO}_{3}$ single crystals reported a spontaneous polarization of $3.5 \mu \mathrm{C} / \mathrm{cm}^{2}$ along the [100] direction (corresponding to $6.1 \mu \mathrm{C} / \mathrm{cm}^{2}$ along the polar [111] axis), but the corresponding hysteresis loops were far from being saturated [39]. Similar small values of the polarization were subsequently reported for $\left(\mathrm{Bi}_{0.7} \mathrm{Ba}_{0.3}\right)\left(\mathrm{Fe}_{0.7} \mathrm{Ti}_{0.3}\right) \mathrm{O}_{3}$ films on Nb-doped $\mathrm{SrTiO}_{3}$ (100) [43] and for polycrystalline $\mathrm{BiFeO}_{3}$ films [44]. However, all these measurements were hampered by the high conductivity of the available samples. 


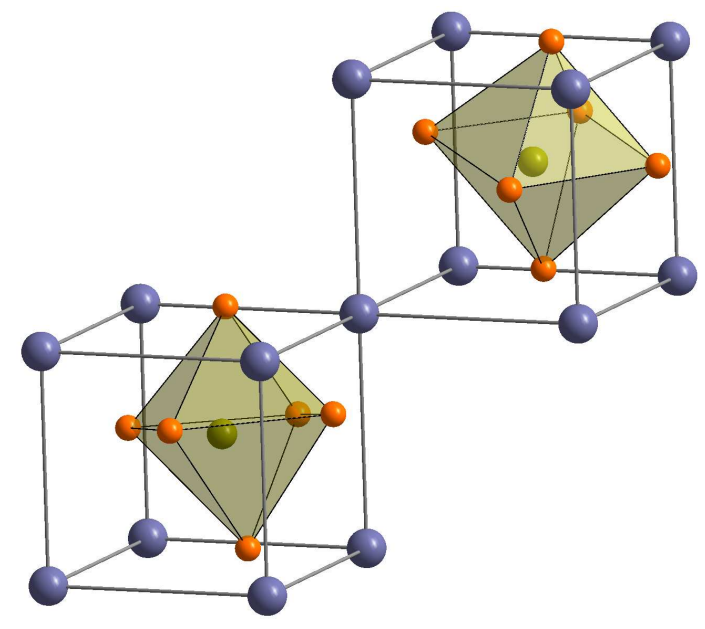

Fig. 3. $\mathrm{BiFeO}_{3}$ crystallizes in a distorted perovskite structure with space group $R 3 c$. The cations are displaced from their ideal positions relative to the anions along the [111] direction. In addition, the oxygen octahedra are rotated around the [111] direction, alternately clockwise and counterclockwise (Bi: blue, Fe: green, O: red).

Interest in $\mathrm{BiFeO}_{3}$ has grown considerably over the last few years, following a report of large electric polarization and substantial thickness-dependent magnetization in epitaxial thin films [7]. The large ferroelectric polarization and macroscopic magnetization were unexpected, since all earlier studies had reported only a small value of the polarization and no magnetization. Therefore the large polarization measured in the epitaxial thin films of $\mathrm{BiFeO}_{3}$ was initially ascribed to the effect of the heteroepitaxial constraint and the resulting change in the lattice parameters. Since a multiferroic with large polarization and magnetization above room temperature would be very desirable for technological applications [12], a flurry of experimental activity ensued but could not resolve the confusion, with a range of reported polarizations ranging between $2.2 \mu \mathrm{C} / \mathrm{cm}^{2}[44]$ and $158 \mu \mathrm{C} / \mathrm{cm}^{2}$ [45].

The spread in magnetization and polarization data therefore posed a number of questions:

(1) What are the intrinsic values of the spontaneous polarization and the magnetization in $\mathrm{BiFeO}_{3}$ ?

(2) How are the polarization and the magnetization in $\mathrm{BiFeO}_{3}$ affected by epitaxial strain?

(3) How are the polarization and magnetization affected by defects?

In the following we show how all these questions have been answered using first-principles calculations. 


\subsubsection{Intrinsic polarization of $\mathrm{BiFeO}_{3}$}

In Ref. [7] the large polarization found in the epitaxial thin films of $\mathrm{BiFeO}_{3}$ was initially ascribed to the effect of the heteroepitaxial constraint and the resulting change in the lattice parameters. To test this hypothesis, the polarization was calculated from first-principles, both in the bulk rhombohedral structure, and a hypothetical tetragonal structure, with its in-plane lattice parameters constrained to that of the substrate. These first calculations seemed to corroborate the hypothesis that the large polarization was due to the epitaxial constraint, since the calculated values for the two different structures were considerably different. However, the LSDA was used in these calculations and no insulating centrosymmetric reference structure (see Section 2) could be obtained. Therefore, only the absolute values of the polarizations were reported (that is the values at $+100 \%$ and $-100 \%$ distortion in Fig. 1) and not the differences in polarization between the ferroelectric structure and a centrosymmetric reference structure, which would correspond to the polarization measured in a ferroelectric switching experiment. Note also, that subsequent experiments showed that the structure in the films is in fact similar to the rhombohedral bulk structure, albeit with an additional monoclinic distortion in the case of a (001) oriented substrate $[46,47,48]$.

Subsequent first-principles calculations using the LSDA $+U$ method were able to obtain an insulating solution for $\mathrm{BiFeO}_{3}$ in the centrosymmetric cubic perovskite structure and in the $R \overline{3} c$ structure, both of which are reasonable reference structures for calculating the spontaneous polarization of $\mathrm{BiFeO}_{3}$ in the rhombohedral bulk structure [19]. Calculation of the polarization change along the so-defined switching path resulted in a value for the spontaneous polarization in bulk $\mathrm{BiFeO}_{3}$ of about $95 \mu \mathrm{C} / \mathrm{cm}^{2}$ along the [111] direction, consistent with the values measured in the thin films [7,46], but exceeding the measured values in the bulk samples by one order of magnitude. This result suggested that the small values of the polarization reported in the past were in fact due to incomplete switching and the resulting unsaturated hysteresis loops represented only a fraction of the true polarization of $\mathrm{BiFeO}_{3}$. The large polarization found in the thin films is then the first really saturated measurement of the full polarization of $\mathrm{BiFeO}_{3}$.

\subsubsection{Effects of strain and defects on the spontaneous polarization in $\mathrm{BiFeO}_{3}$}

It is known from materials such as $\mathrm{BaTiO}_{3}$ and $\mathrm{SrTiO}_{3}$ that the epitaxial strain present in thin film samples can lead to drastic changes in the ferroelectric polarization compared to the corresponding bulk materials [49,50]. The good agreement between the calculated bulk value of the polarization and the experimentally observed thin film value in $\mathrm{BiFeO}_{3}$ therefore seems to be in

conflict with the strong strain dependence of polarization expected in general 
for ferroelectrics. The lattice mismatch between $\mathrm{BiFeO}_{3}$ and $\mathrm{SrTiO}_{3}$, the substrate material used in Ref. [7], amounts to about $2 \%$ compressive strain in the $\mathrm{BiFeO}_{3}$ film, which is a substantial value.

To address the epitaxial strain dependence of the ferroelectric polarization in rhombohedral $\mathrm{BiFeO}_{3}$ a detailed first-principles study was carried out. Epitaxial strain was imposed by constraining the in-plane lattice parameters over a range of non-equilibrium values, and allowing the structure to relax out-ofplane at each constraint [51]. In contrast to similar calculations for $\mathrm{BaTiO}_{3}$ and $\mathrm{PbTiO}_{3}[52,53,54]$, it was found that the electric polarization in $\mathrm{BiFeO}_{3}$ is not significantly affected by the presence of epitaxial strain. Even strain values as large as $\pm 3 \%$ lead only to changes in the polarization of a few percent, provided that the material does not undergo a structural phase transition. (For comparison, the corresponding change in $\mathrm{BaTiO}_{3}$ is more than $100 \%$ [52]). The relative strain-independence of the polarization in $\mathrm{BiFeO}_{3}$ supports the notion that the large polarization found in the thin films is essentially equal to the bulk value and that the old measurements severely underestimated the polarization in $\mathrm{BiFeO}_{3}$.

Recently, possible reasons for this different strain behavior between $\mathrm{BiFeO}_{3}$ and ferroelectrics like $\mathrm{BaTiO}_{3}$ or $\mathrm{PbTiO}_{3}$ have been addressed using firstprinciples methods [55]. It was shown that the variation of the spontaneous polarization with epitaxial strain in thin film ferroelectrics can be understood in terms of the piezoelectric and elastic constants of the corresponding bulk materials, and that a large strain dependence is not a common feature of all ferroelectrics.

Ref. [51] also investigated the effect of oxygen vacancies on the spontaneous polarization in $\mathrm{BiFeO}_{3}$ using first-principles techniques. It was shown that the incorporation of such vacancies leads to small relaxations around the vacancy site and to the formation of $\mathrm{Fe}^{2+}$ next to the vacancy site, but that the value of the spontaneous polarization is not significantly affected by the presence of oxygen vacancies.

\subsubsection{Magnetic properties of $\mathrm{BiFeO}_{3}$}

First-principles calculations have also been applied to understanding the magnetic properties of $\mathrm{BiFeO}_{3}$, in particular the observation of magnetization in $\mathrm{BiFeO}_{3}$ thin films and its absence in bulk samples. One possible source of magnetization in a primarily antiferromagnetic material is weak ferromagnetism, i.e. a canting of the mainly antiferromagnetically oriented magnetic moments resulting in a small magnetization [56]. In bulk $\mathrm{BiFeO}_{3}$, the G-type antiferromagnetic ordering [41] is modulated by a long wavelength spiral spin structure [42] which would lead to a cancellation of the magnetization on a macroscopic 
scale. However, it was suggested that the thin film morphology might suppress the spiral spin structure so that the weak ferromagnetism becomes observable in thin films [56,57]. First-principles calculations indeed showed that a local canting does occur, and that its origin is the Dzyaloshinskii-Moriya interaction [58]. The magnitude of the calculated value for the magnetization is approximately $0.1 \mu_{\mathrm{B}} /$ (unit cell), in good agreement with recent measurements $[57,59]$. Therefore, this supports the interpretation proposed above for the origin of the magnetization in thin films of $\mathrm{BiFeO}_{3}$.

The thin film measurements of Ref. [7] also reported a strong increase in magnetization with decreasing film thickness, reaching values of about $1 \mu_{\mathrm{B}} / \mathrm{Fe}$ for film thicknesses below $100 \mathrm{~nm}$. Since neither calculations on ideal systems [56] nor recent measurements on highly stoichiometric $\mathrm{BiFeO}_{3}$ films [59] were able to reproduce an increased magnetization, it was suggested that oxygen vacancies and the resulting presence of $\mathrm{Fe}^{2+}$ could lead to the observed increase in the magnetization of very thin films [60]. However, supercell calculations corresponding to different concentrations of oxygen vacancies were not able to confirm this suggestion [51]. In these calculations it was found that removing a neutral oxygen atom from the supercell resulted in the formation of $\mathrm{Fe}^{2+}$ on the sites adjacent to the vacancy site. The magnetic moments of such a pair of $\mathrm{Fe}^{2+}$ cations are coupled antiferromagnetically and therefore do not result in a net magnetization. Also, the canting of the magnetic moments was not significantly affected by the presence of the oxygen vacancies, so that no increase in the macroscopic magnetization due to oxygen vacancies could be found. Ref. [51] also showed that the canting, and with this the magnetization, is not significantly enhanced by epitaxial strain. The reason for the increase in magnetization with decreasing film thickness in Ref. [7] is therefore still unclear, but from the first-principles calculations it can be concluded that the observed effect must be caused by effects other than epitaxial strain or neutral oxygen vacancies.

\subsection{Other systems}

Very recently, phonon energies were calculated for the spinel system $\mathrm{CdCr}_{2} \mathrm{~S}_{4}$ [61]. This material, which is known to be a rare example of a ferromagnetic insulator with a rather high magnetic Curie temperature, was recently reported to exhibit interesting coupling between dielectric and magnetic properties as well as a small electric polarization at low temperatures [62]. It was suggested that the relaxor-like behavior could be due to frustrated soft-mode behavior and that the ferroelectricity at low-temperatures could originate from a displacement of the $\mathrm{Cr}^{3+}$ ion [62]. The first-principles calculation on the other hand showed that all phonon modes in this material are rather stable and that the scenario of a $\mathrm{Cr}^{3+}$ ion moving off-center is extremely unlikely [61]. More 
recent experimental results indicate that the observed behavior is probably defect-related and not an intrinsic effect since the observed features in the dielectric constant vanish after annealing the corresponding samples [63]. This represents another example how first-principles calculations can be used to separate intrinsic material properties from extrinsic effects like microstructure or defects.

\subsection{Summary of first-principles explanation of experimental observations}

It is clear from the above examples that first-principles calculations have made many contributions to our understanding of multiferroic phenomena, and that the interplay between theory and experiment has been invaluable for progress in the field. The example of $\mathrm{YMnO}_{3}$ showed how the mechanism underlying unconventional ferroelectric phase transitions can be elucidated using firstprinciples techniques, and the example of $\mathrm{BiFeO}_{3}$ showed how first-principles calculations can be used for resolving conflicting experimental results and to separate intrinsic materials properties from extrinsic effects such as defects or microstructure.

\section{First-principles predictions of new materials and novel phenom- ena}

In this section we change our focus compared to the previous section and discuss examples where predictions have been made using first-principles techniques prior to their experimental verification.

\subsection{Electric-field induced magnetization switching}

The most important question arising from the presence of both spontaneous electric polarization and macroscopic magnetization in a multiferroic is whether these two properties are coupled, and, if so, in what manner. A coupling that facilitates the reversal of the magnetization by an electric field or conversely, the reversal of the electric polarization by a magnetic field, would be of particular interest. In the following we show how first-principles calculations have been used to study this question, and to develop theoretical models for coupling between magnetic and structural/dielectric properties.

Again, $\mathrm{BiFeO}_{3}$ has been used as the prototype material for first-principles

study. Calculations of the coupling between magnetism and structural distortions in $\mathrm{BiFeO}_{3}$ showed that the Dzyaloshinskii-Moriya interaction [58], which 


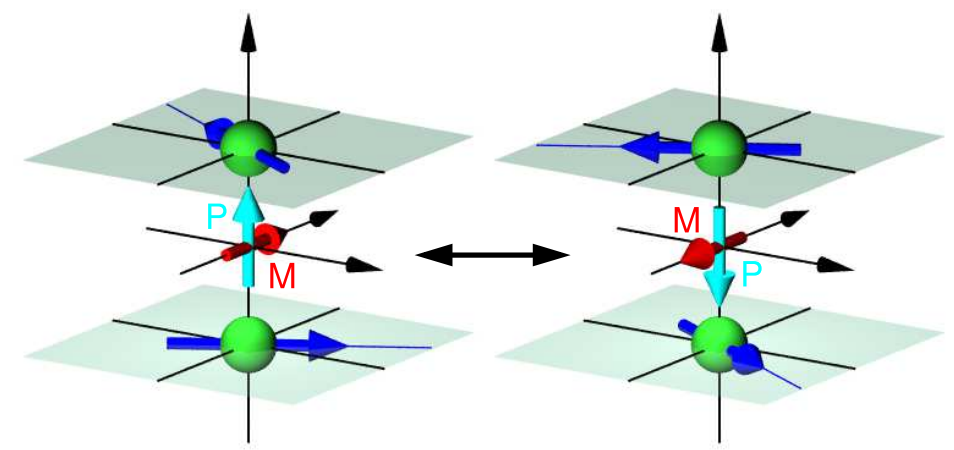

Fig. 4. Possible realization of electric-field induced magnetization switching in a weak ferromagnetic ferroelectric: if the polarization $P$ is reversed, the canting of the antiferromagnetic sublattices, and with this the magnetization $M$, is also reversed.

leads to the canting of the magnetic moments and the appearance of a macroscopic magnetization, is indeed a result of the symmetry lowering structural distortions in this material (in the high symmetry cubic phase, weak ferromagnetism is not allowed by symmetry) [56]. In the case of $\mathrm{BiFeO}_{3}$ there are two different symmetry lowering distortions that together reduce the symmetry from the cubic perovskite structure $(P m \overline{3} m)$ to the ferroelectric rhombohedral ground state structure $(R 3 c)$. One is a nonpolar $R$ mode that leads to a unit cell doubling compared to the simple perovskite structure and rotations of the oxygen octahedra around the rhombohedral axis, and the other is a polar $\Gamma$ mode that displaces all ions relative to each other along the same rhombohedral axis (see Figure 3). It was shown that the Dzyaloshinskii-Moriya interaction in $\mathrm{BiFeO}_{3}$ is caused by the nonpolar mode and that reversal of that mode also inverts the magnetization direction [56].

This has important consequences for the possible realization of electric-fieldinduced magnetization switching, which was believed to be impossible due to symmetry reasons [64]. From the results obtained in Ref. [56] it became clear that such an effect is in principle possible in weak ferromagnetic systems in which the polar distortion, which results in ferroelectricity, also leads to the appearance of a Dzyaloshinskii-Moriya interaction, which results in weak ferromagnetism. In such a scenario, the magnetization will be strongly coupled to the ferroelectric polarization and reversal of the ferroelectric polarization by an electric field will be accompanied by reversal of the macroscopic magnetization [56] (see Figure 4). The search for a material where this effect can be realized is a topic of current research activity [65]. 


\subsection{Computational materials design}

The examples discussed in the previous sections illustrate that first-principles calculations are capable of correctly predicting numerous properties of magnetoelectric multiferroics. This opens up the possibility of $a b$ initio computational design of entirely new multiferroic materials, with improved properties, in advance of their synthesis. Such a materials design approach was suggested in Ref. [66] and has been applied since then to search for new multiferroics with properties superior to those of existing materials.

The scarcity of magnetoelectric multiferroics has rendered such an approach extremely desirable. For example, most magnetoelectric multiferroics are either antiferromagnets or exhibit only weak ferromagnetism, often below room temperature. On the other hand, for technological applications a multiferroic with large polarization and large magnetization, well above room temperature, and preferably with strong coupling between the two properties is needed. Currently, no magnetoelectric material is known that fulfills all these requirements. Since the calculation of the properties of a variety of candidate materials is often less costly than the corresponding experimental synthesis and subsequent characterization, a first-principles design approach is very appealing.

Probably the first example for the ab initio design of a magnetoelectric multiferroic was the prediction of ferroelectricity in $\mathrm{BiMnO}_{3}$. This material was known to be ferromagnetic at low temperatures [67], and the calculation of unstable zone-center phonon modes in cubic $\mathrm{BiMnO}_{3}$ suggested the simultaneous existence of ferroelectricity [68]. Motivated by this prediction, experimental investigations were performed and ferroelectric hysteresis loops were reported for polycrystalline samples as well as for thin films of $\mathrm{BiMnO}_{3}$ [69]. The ferromagnetism in $\mathrm{BiMnO}_{3}$ is interesting in its own right, and is believed to be due to orbital ordering caused by a cooperative Jahn-Teller distortion [70]. Such an orbital ordering was reproduced recently by first-principles calculations using the experimentally obtained structure [71].

A first-principles search for multiferroism has also been performed for the closely related compound $\mathrm{BiCrO}_{3}[72]$. Prior to the theoretical study, little was known experimentally about the material; early papers reported antiferromagnetism with a weak ferromagnetic moment below $123 \mathrm{~K}$, and a structural transition between pseudomonoclinic and pseudotriclinic perovskite structures at 400K [73]. The first-principles calculations found an antiferromagnetic ground state, in agreement with the Goodenough/Kanamori/Anderson rules for superexchange interactions in magnetic insulators [74,75], and the calculation of the full phonon spectrum revealed several unstable phonon branches over the whole range of the Brillouin zone. The most unstable modes were found at the $R$ and $M$ points of the Brillouin zone corresponding to antiferroelec- 
tric or antiferrodistortive phonon modes. Since the antiferromagnetic ordering in $\mathrm{BiCrO}_{3}$ does not lead to a macroscopic magnetization, it was concluded that this material will not be of great technological relevance and a full structural optimization of the ground state structure was not attempted [72]. A subsequent experimental study [76] confirmed the weak ferromagnetism, and observed a non-polar to polar structural phase transition, suggestive of ferroelectricity, at $440 \mathrm{~K}$.

The difficulty of incorporating a substantial magnetization in a magnetoelectric multiferroic results from the dominance of magnetic superexchange in such transition metal oxides, which in most cases leads to antiferromagnetic nearest neighbor coupling. Although in certain cases the superexchange mechanism can lead to ferromagnetic coupling, the strength of such a ferromagnetic coupling is usually substantially weaker than in the antiferromagnetic case [75], resulting in low magnetic ordering temperatures. Therefore a recent proposal to exploit the strong antiferromagnetic superexchange and introduce macroscopic magnetization by combining two magnetic ions with different magnetic moments, resulting in a ferrimagnetic spin-arrangement [77] is very promising. The ordered double perovskite system $\mathrm{Bi}_{2} \mathrm{FeCrO}_{6}$ was suggested as a candidate material, since the stereochemically active $\mathrm{Bi}^{3+}$ ion should introduce a potentially ferroelectric structural distortion and the closed-subshell $d^{5} \mathrm{Fe}^{3+}$ and $d^{3} \mathrm{Cr}^{3+}$ ions should result in insulating behavior.

Indeed, a ferroelectric ground state with space group $R 3$ was found by structural optimization using first-principles calculations within the LSDA $+U$ method [77]. The corresponding structure is closely related to the $R 3 c$ structure found in the well-known multiferroic $\mathrm{BiFeO}_{3}$ [19] and is also consistent with the strong phonon instability at the $R$ point found in cubic $\mathrm{BiCrO}_{3}$ [72]. A rocksalt-like ordering of $\mathrm{Fe}^{3+}$ and $\mathrm{Cr}^{3+}$ cations on the $B$ site lattice of the perovskite structure was enforced in the calculation; such ordered structures can be achieved by layer-by-layer growth in epitaxial films, as has been demonstrated experimentally for the case of $\mathrm{La}_{2} \mathrm{FeCrO}_{6}$ [78]. The calculated spontaneous electric polarization of $\mathrm{Bi}_{2} \mathrm{FeCrO}_{6}$ was about $80 \mu \mathrm{C} / \mathrm{cm}^{2}$. The calculated ground state magnetic structure was the ferrimagnetic equivalent of G-type antiferromagnetic ordering, with oppositely oriented magnetic moments of the $\mathrm{Fe}^{3+}$ and $\mathrm{Cr}^{3+}$ ions resulting in a magnetization of $2 \mu_{\mathrm{B}}$ /formula unit. Thus, ordered $\mathrm{Bi}_{2} \mathrm{FeCrO}_{6}$ was predicted to be a unique magnetoelectric multiferroic with large electric polarization and large magnetization [77]. The magnetic ordering temperature of the newly predicted magnetoelectric multiferroic $\mathrm{Bi}_{2} \mathrm{FeCrO}_{6}$, estimated by calculating the nearest and next-nearest neighbor coupling constants from first-principles and then applying the mean-field approximation, was shown to not exceed $100 \mathrm{~K}$ [79]. Since this mean-field value can be viewed as an upper bound for the real magnetic ordering temperature it is unlikely that $\mathrm{Bi}_{2} \mathrm{FeCrO}_{6}$ will show multiferroic behavior at room temperature. 
In order to gain more insight into the factors that promote high magnetic ordering temperatures in such ferromagnetic ferroelectrics, a comparative study of the series of multiferroics $\mathrm{BiFeO}_{3}, \mathrm{Bi}_{2} \mathrm{FeCrO}_{6}$, and $\mathrm{BiCrO}_{3}$ was performed [79]. The magnetic ordering temperatures of the different compounds were again obtained by calculating the nearest and next nearest neighbor exchange coupling constants using first principles methods and determining the resulting ordering temperature within the mean-field approximation. It was found that the calculated variation of the strength of the nearest neighbor coupling constants could be explained based on the different electron configurations of the constituent ions using Anderson's theory of superexchange [75]. In addition, the influence of the structural distortions on the magnetic coupling was determined by comparing the calculated coupling constants for the relaxed ferroelectric structures with those obtained for the corresponding undistorted cubic structures. In general, the structural distortions resulted in a weakening of the antiferromagnetic coupling, and in the case of $\mathrm{Bi}_{2} \mathrm{FeCrO}_{6}$ an interesting crossover behavior from antiferromagnetic to ferromagnetic nearest neighbor coupling could be observed, again in good agreement with the qualitative predictions from the theory of superexchange [79]. The insight gained by studying the systematic variations of exchange interactions in this series of compounds was used to propose a variety of other systems with potentially larger magnetic coupling. Since the theory of superexchange is only able to give qualitative predictions, however, additional first-principles calculations will be necessary to make quantitative predictions.

\section{Summary and Outlook}

From the examples discussed in the present paper it is clear that first principles methods are very powerful tools for studying multiferroic materials and magnetoelectric coupling. These methods facilitate the accurate quantitative calculation of many material properties and can therefore be used to establish benchmark values to verify experimentally obtained data. Furthermore, the detailed analysis of the calculated results and the possibility to perfectly control the system under investigation allows the development of new theoretical concepts and the construction of phenomenological models to gain a deeper understanding of the basic physics behind multiferroic phenomena. This knowledge can then be used to design new materials with desirable properties and specific values can be calculated explicitly for various candidate materials. The examples discussed in the previous sections illustrate how first-principles calculations have been used to deliver benchmark data for the magnetization and polarization in $\mathrm{BiFeO}_{3}$, to understand the mechanism underlying the ferroelectricity in $\mathrm{YMnO}_{3}$, to establish the concept of electric-field induced magnetization switching in weak ferromagnets, and to design new multiferroic 
materials with formerly unachievable properties.

In the future, first-principles methods will undoubtedly continue to make invaluable contributions to the field of magnetoelectric multiferroics. For example, efforts are underway to describe effects of spin-phonon coupling [80], and to identify a material that exhibits electric-field switchable weak ferromagnetism [65]. In addition, new classes of multiferroic materials remain to be explored, for example the orthorhombic and hexagonal rare earth manganites, which show a variety of interesting coupling effects between magnetic and ferroelectric properties $[8,11]$. Nanostructured magnetoelectric composites [81] offer a new way of coupling magnetic and ferro- or piezoelectric properties, and the multitude of possible materials combinations represents a way to tune the properties of the heterostructure to the desired values. Again, first-principles calculations offer a powerful tool to study the properties of such heterostructures and to predict the characteristics of different materials combinations. We therefore believe that the first-principles study of multiferroic materials will continue to be very exciting and that these methods will continue to contribute significantly to the progress in this field.

\section{References}

[1] S. M. Skinner, Magnetically ordered ferroelectric materials, IEEE Trans. Parts, Materials and Packaging PMP-6 (1970) 68-90.

[2] H. Schmid, On a magnetoelectric classification of materials, Int. J. Magnetism 4 (1973) 337-361.

[3] G. A. Smolenskii, I. E. Chupis, Ferroelectromagnets, Sov. Phys. Usp. 25 (1982) 475-493.

[4] H. Schmid, Multi-ferroic magnetoelectrics, Ferroelectrics 62 (1994) 317.

[5] N. A. Hill, Why are there so few magnetic ferroelectrics?, J. Phys. Chem. B 104 (2000) 6694.

[6] M. Fiebig, T. Lottermoser, D. Fröhlich, A. V. Goltsev, R. V. Pisarev, Observation of coupled magnetic and electric domains, Nature (London) 419 (2002) 818-820.

[7] J. Wang, J. B. Neaton, H. Zheng, V. Nagarajan, S. B. Ogale, B. Liu, D. Viehland, V. Vaithyanathan, D. G. Schlom, U. V. Waghmare, N. A. Spaldin, K. M. Rabe, M. Wuttig, R. Ramesh, Epitaxial $\mathrm{BiFeO}_{3}$ multiferroic thin film heterostructures, Science 299 (2003) 1719.

[8] T. Kimura, T. Goto, H. Shintani, K. Ishizaka, T. Arima, Y. Tokura, Magnetic control of ferroelectric polarization, Nature (London) 426 (2003) 55-58. 
[9] T. Kimura, S. Kawamoto, I. Yamada, M. Azuma, M. Takano, Y. Tokura, Magnetocapacitance effect in multiferroic $\mathrm{BiMnO}_{3}$, Phys. Rev. B 67 (2003) 180401(R).

[10] N. Hur, S. Park, P. A. Sharma, J. S. Ahn, S. Guha, S.-W. Cheong, Electric polarization reversal and memory in a multiferroic material induced by magnetic fields, Science 429 (2004) 392-395.

[11] T. Lottermoser, T. Lonkai, U. Amann, D. Hohlwein, J. Ihringer, M. Fiebig, Magnetic phase control by an electric field, Nature (London) 430 (2004) 541544.

[12] M. Fiebig, Revival of the magnetoelectric effect, J. Phys. D: Appl. Phys. 38 (2005) R1-R30.

[13] N. A. Hill, Density functional studies of multiferroic magnetoelectrics, Annu. Rev. Mater. Res. 32 (2002) 1-37.

[14] R. E. Cohen, Origin of ferroelectricity in perovskite oxides, Nature 358 (1992) $136-138$.

[15] R. E. Cohen, Theory of ferroelectrics: a vision for the next decade and beyond, J. Phys. Chem. Solids 61 (2000) 139-146.

[16] A. Filippetti, N. A. Hill, Coexistence of magnetism and ferroelectricity in perovskites, Phys. Rev. B 65 (2002) 195120.

[17] N. A. Hill, A. Filippetti, Why are there any magnetic ferroelectrics?, J. Magn. Magn. Mater. 242-245 (2002) 976-979.

[18] R. Seshadri, N. A. Hill, Visualizing the role of Bi $6 s$ "lone pairs" in the off-center distortion in ferromagnetic $\mathrm{BiMnO}_{3}$, Chem. Mater. 13 (2001) 2892-2899.

[19] J. B. Neaton, C. Ederer, U. V. Waghmare, N. A. Spaldin, K. M. Rabe, Firstprinciples study of spontaneous polarization in multiferroic $\mathrm{BiFeO}_{3}$, Phys. Rev. B 71 (2005) 014113.

[20] R. O. Jones, O. Gunnarsson, The density functional formalism, its applications and prospects, Rev. Mod. Phys. 61 (1989) 689-746.

[21] R. M. Martin, Electronic Structure, Cambridge University Press, 2004.

[22] K. Terakura, T. Oguchi, A. R. Williams, J. Kübler, Band theory of insulating transition-metal monoxides: Band-structure calculations, Phys. Rev. B 30 (1984) 4734-4747.

[23] P. Ghosez, J.-P. Michenaud, X. Gonze, Dynamical atomic charges: The case of $\mathrm{abo}_{3}$ compounds, Phys. Rev. B 58 (1998) 6224-6239.

[24] V. I. Anisimov, F. Aryasetiawan, A. I. Liechtenstein, First-principles calculations of the electronic structure and spectra of strongly correlated systems: the LDA $+U$ method, J. Phys.: Condens. Matter 9 (1997) 767-808. 
[25] A. Filippetti, N. A. Spaldin, Self-interaction-corrected pseudopotential scheme for magnetic and strongly-correlated systems, Phys. Rev. B 67 (2003) 125109.

[26] J. P. Perdew, A. Zunger, Self-interaction correction to density-functional approximations for many-electron systems, Phys. Rev. B 23 (1981) 5048-5078.

[27] V. I. Anisimov, J. Zaanen, O. K. Anderson, Band theory and Mott insulators: Hubbard $U$ instead of Stoner I, Phys. Rev. B 44 (1991) 943-954.

[28] I. V. Solovyev, P. H. Dederichs, V. I. Anisimov, Corrected atomic limit in the local-density approximation and the electronic structure of $d$ impurities in Rb, Phys. Rev. B 50 (1994) 16861-16871.

[29] R. D. King-Smith, D. Vanderbilt, Theory of polarization of crystalline solids, Phys. Rev. B 47 (1993) R1651-R1654.

[30] D. Vanderbilt, R. D. King-Smith, Electric polarization as a bulk quantity and its relation to surface charges, Phys. Rev. B 48 (1993) 4442-4455.

[31] R. Resta, Macroscopic polarization in crystalline dielectrics: the geometric phase approach, Rev. Mod. Phys. 66 (1994) 899-915.

[32] H. L. Yakel, W. C. Koehler, E. F. Bertaut, E. F. Forrat, On the crystal structure of the manganese (III) trioxides of the heavy lanthanides and yttrium, Acta Cryst. 16 (1963) 957-962.

[33] J. E. Medvedeva, V. I. Anisimov, M. A. Korotin, O. N. Mryasov, A. J. Freeman, The effect of coulomb correlation and magnetic ordering on the electronic structure of two hexagonal phases of ferroelectromagnetic $\mathrm{YMnO}_{3}$, J. Phys.: Condens. Matter 12 (2000) 4947-4958.

[34] M. Qian, J. Dong, Q. Zheng, Electronic structure of the ferroelectromagnet $\mathrm{YMnO}_{3}$, Physics Letters A 270 (2000) 96-101.

[35] A. Filippetti, N. A. Hill, First principles study of structural, electronic and magnetic interplay in ferroelectromagnetic yttrium manganite, J. Magn. Magn. Mater. 236 (2001) 176-189.

[36] B. B. van Aken, A. Meetsma, T. T. M. Palstra, Hexagonal $\mathrm{YMnO}_{3}$, Acta Cryst. C57 (2001) 230-232.

[37] B. B. van Aken, T. T. M. Palstra, A. Filippetti, N. A. Spaldin, The origin of ferroelectricity in magnetoelectric $\mathrm{YMnO}_{3}$, Nature Materials 3 (2004) 164.

[38] C. J. Fennie, K. M. Rabe, Ferroelectric transition in $\mathrm{YMnO}_{3}$ from first principles, Phys. Rev. B 72 (2005) 100103(R).

[39] J. R. Teague, R. Gerson, W. J. James, Dielectric hysteresis in single crystal $\mathrm{BiFeO}_{3}$, Solid State Commun. 8 (1970) 1073-1074.

[40] S. V. Kiselev, R. P. Ozerov, G. S. Zhdanov, Detection of magnetic order in ferroelectric $\mathrm{BiFeO}_{3}$ by neutron diffraction, Sov. Phys. Dokl. 7 (1963) 742-744. 
[41] P. Fischer, M. Polemska, I. Sosnowska, M. Szymański, Temperature dependence of the crystal and magnetic structure of $\mathrm{BiFeO}_{3}$, J. Phys. C 13 (1980) 19311940 .

[42] I. Sosnowska, T. Peterlin-Neumaier, E. Streichele, Spiral magnetic ordering in bismuth ferrite, J. Phys. C 15 (1982) 4835-4846.

[43] K. Ueda, H. Tabata, T. Kawai, Coexistence of ferroelectricity and ferromagnetism in $\mathrm{BiFeO}_{3}-\mathrm{BaTiO}_{3}$ thin films at room temperature, Appl. Phys. Lett. 75 (1999) $555-557$.

[44] V. R. Palkar, J. John, R. Pinto, Observation of saturated polarization and dielectric anomaly in magnetoelectric $\mathrm{BiFeO}_{3}$ thin films, Appl. Phys. Lett. 80 (2002) 1628.

[45] K. Y. Yun, D. Ricinschi, T. Kanashima, M. Noda, M. Okuyama, Giant ferroelectric polarization beyond $150 \mu \mathrm{c} / \mathrm{cm}^{2}$ in $\mathrm{BiFeO}_{3}$ thin films, Jpn. J. Appl. Phys. 43 (2004) L647.

[46] J. Li, J. Wang, M. Wuttig, R. Ramesh, N. Wang, B. Ruette, A. P. Pyatakov, A. K. Zvezdin, D. Viehland, Dramatically enhanced polarization in (001), (101), and (111) $\mathrm{BiFeO}_{3}$ thin films due to epitaxial-induced transitions, Appl. Phys. Lett. 84 (2004) 5261-5263.

[47] X. Qi, M. Wei, Y. Lin, Q. Jia, D. Zhi, J. Dho, M. G. Blamire, J. L. MacManusDriscoll, High resolution x-ray diffraction and transmission electron microscopy of multiferroic $\mathrm{BiFeO}_{3}$, Appl. Phys. Lett. 86 (2005) 071913.

[48] G. Xu, H. Hiraka, G. Shirane, J. Li, J. Wang, D. Viehland, Low symmetry phase in (001) $\mathrm{BiFeO}_{3}$ epitaxial constrained thin films, Appl. Phys. Lett. 86 (2005) 182905 .

[49] K. J. Choi, M. Biegalski, Y. L. Li, A. Sharan, J. Schubert, R. Uecker, P. Reiche, Y. B. Chen, X. Q. Pan, V. Gopalan, L.-Q. Chen, D. G. Schlom, C. B. Eom, Enhancement of ferroelectricity in strained $\mathrm{BaTiO}_{3}$ thin films, Science 306 (2004) 1005-1009.

[50] J. H. Haeni, P. Irvin, W. Chang, R.Uecker, P. Reiche, Y. L. Li, S. Choudhury, W. Tian, M. E. Hawley, B. Craigo, A. K. Tagantsev, X. Q. Pan, S. K. Streiffer, L. Q. Chen, S. W. Kirchoefer, J. Levy, D. G. Schlom, Room-temperature ferroelectricity in strained $\mathrm{SrTiO}_{3}$, Nature (London) 430 (2004) 758-761.

[51] C. Ederer, N. A. Spaldin, Influence of strain and oxygen vacancies on the magnetoelectric properties of multiferroic bismuth ferrite, Phys. Rev. B 71 (2005) 224103.

[52] J. B. Neaton, C.-L. Hsueh, K. M. Rabe, Enhanced polarization in strained $\mathrm{BaTiO}_{3}$ from first principles, cond-mat/0204511.

[53] J. B. Neaton, K. M. Rabe, Theory of polarization enhancement in epitaxial $\mathrm{BaTiO}_{3} / \mathrm{SrTiO}_{3}$ superlattices, Appl. Phys. Lett. 82 (2003) 1586-1588. 
[54] C. Bungaro, K. M. Rabe, Epitaxially strained [001]- $\left(\mathrm{PbTiO}_{3}\right)_{1}\left(\mathrm{PbZrO}_{3}\right)_{1}$ superlattice and $\mathrm{PbTiO}_{3}$ from first principles, Phys. Rev. B 69 (2004) 184101.

[55] C. Ederer, N. A. Spaldin, Effect of epitaxial strain on the spontaneous polarization of thin film ferroelectrics, cond-mat/0508005.

[56] C. Ederer, N. A. Spaldin, Weak ferromagnetism and magnetoelectric coupling in bismuth ferrite, Phys. Rev. B 71 (2005) 060401.

[57] F. Bai, J. Wang, M. Wuttig, J. Li, N. Wang, A. P. P. dn A. K. Zvezdin, L. E. Cross, D. Viehland, Destruction of spin cycloid in $(111)_{c}$-oriented $\mathrm{BiFeO}_{3}$ thin films by epitaxial constraint: Enhanced polarization and release of latent magnetization, Appl. Phys. Lett. 86 (2005) 032511.

[58] T. Moriya, Weak ferromagnetism, in: G. T. Rado, H. Suhl (Eds.), Magnetism, Vol. 1, Academic Press, 1963, Ch. 3, pp. 85-125.

[59] W. Eerenstein, F. D. Morrison, J. Dho, M. G. Blamire, J. F. Scott, N. Mathur, Comment on "Epitaxial $\mathrm{BiFeO}_{3}$ multiferroic thin film heterostructures", Science 307 (2005) 1203a.

[60] J. Wang, A. Scholl, H. Zheng, S. B. Ogale, D. Viehland, D. G. Schlom, N. A. Spaldin, K. M. Rabe, M. Wuttig, L. Mohaddes, J. Neaton, U. Waghmare, T. Zhao, R. Ramesh, Response to comment on "Epitaxial $\mathrm{BiFeO}_{3}$ multiferroic thin film heterostructures", Science 307 (2005) 1203b.

[61] C. J. Fennie, K. M. Rabe, Polar phonons and dielectric response of the ferromagnetic insulating spinel $\mathrm{CdCr}_{2} \mathrm{~S}_{4}$ from first principles, condmat/0508135.

[62] J. Hemberger, P. Lunkenheimer, R. Fichtl, H. A. K. von Nidda, V. Tsurkan, A. Loidl, Relaxor ferroelectricity and colossal in ferromagnetic $\mathrm{CdCr}_{2} \mathrm{~S}_{4}$, Nature 434 (2005) 362-367.

[63] J. Hemberger, P. Lunkenheimer, R. Fichtl, S. Weber, V. Tsurkan, A. Loidl, Multiferroic behavior in $\mathrm{CdCr}_{2} X_{4}(X=\mathrm{S}$, Se), cond-mat/050801.

[64] H. Schmid, On the possibility of ferromagnetic, antiferromagnetic, ferroelectric, and ferroelastic domain reorientations in magnetic and electric fields, Ferroelectrics 221 (1999) 9-17.

[65] C. Ederer, N. A. Spaldin, in preparation.

[66] N. A. Spaldin, W. E. Pickett, Computational design of multifunctional materials, J. Solid State Chemistry 176 (2003) 615-632.

[67] V. A. Bokov, I. E. Myl'nikova, S. A. Kizhaev, M. F. Bryzhina, N. A. Grigorian, Structure and magnetic properties of $\mathrm{BiMnO}_{3}$, Sov. Phys. Solid State 7 (1966) 2993-2994.

[68] N. A. Hill, K. M. Rabe, First-principles investigation of ferromagnetism and ferroelectricity in bismuth manganite, Phys. Rev. B 59 (1999) 8759-8769. 
[69] A. M. dos Santos, S. Parashar, A. R. Raju, Y. S. Zhao, A. K. Cheetham, C. N. R. Rao, Evidence for the likely occurence of magnetoferroelectricity in the simple perovskite $\mathrm{BiMnO}_{3}$, Solid State Commun. 122 (2002) 49-52.

[70] A. M. dos Santos, A. K. Cheetham, T. Atou, Y. Syono, Y. Yamaguchi, K. Ohoyama, H. Chiba, C. N. R. Rao, Orbital ordering as the determinant for ferromagnetism in biferroic $\mathrm{BiMnO}_{3}$, Phys. Rev. B 66 (2002) 064425.

[71] T. Shishidou, N. Mikamo, Y. Uratani, F.Ishii, T. Oguchi, First-principles study on the electronic structure of bismuth transition metal oxides, J. Phys.: Condens. Matter 16 (2004) S5677-S5683.

[72] N. A. Hill, P. Bättig, C. Daul, First principles search for multiferroism in $\mathrm{BiCrO}_{3}$, J. Phys. Chem. B 106 (2002) 3383-3388.

[73] F. Sugawara, S. Ida, Y. Syono, S. J. Akimoto, Magnetic properties and crystal distortions of $\mathrm{BiMnO}_{3}$ and $\mathrm{BiCrO}_{3}$, J. Phys. Soc. Jpn. 25 (1968) 1553.

[74] J. B. Goodenough, Magnetism and the Chemical Bond, Interscience Publishers, New York, 1963.

[75] P. W. Anderson, Exchange in insulators: Superexchange, direct exchange, and double exchange, in: G. T. Rado, H. Suhl (Eds.), Magnetism, Vol. 1, Academic Press, 1963, Ch. 2, pp. 25-83.

[76] S. Niitaka, M. Azuma, M. Takano, E. Nishibori, M. Takata, M. Sakata, Crystal structure and dielectric and magnetic properties of $\mathrm{BiCrO}_{3}$ as a ferroelectromagnet, Solid State Ionics 172 (2004) 557-559.

[77] P. Baettig, N. A. Spaldin, Ab initio prediction of a multiferroic with large polarization and magnetization, Appl. Phys. Lett. 86 (2005) 012505.

[78] K. Ueda, H. Tabata, T. Kawai, Ferromagnetism in $\mathrm{LaFeO}_{3}-\mathrm{LaCrO}_{3}$ superlattices, Science 280 (1998) 1064-1066.

[79] P. Baettig, C. Ederer, N. A. Spaldin, First principles study of the multiferroics $\mathrm{BiFeO}_{3}, \mathrm{Bi}_{2} \mathrm{FeCrO}_{6}$, and $\mathrm{BiCrO}_{3}$ : Structure, polarization, and magnetic ordering temperature, Phys. Rev. B 72 (2005) 214105.

[80] C. J. Fennie, K. M. Rabe, in preparation.

[81] H. Zheng, J. Wang, S. E. Lofland, Z. Ma, L. Mohaddes-Ardabili, T. Zhao, L. Salamanca-Riba, S. R. Shinde, S. B. Ogale, F. Bai, D. Viehland, Y. Jia, D. G. Schlom, M. Wuttig, A. Roytburd, R. Ramesh, Multiferroic $\mathrm{BaTiO}_{3}-\mathrm{CoFe}_{2} \mathrm{O}_{4}$ nanostructures, Science 303 (2004) 661-663. 